\title{
Continuous Cardiac Stroke Volume Monitoring Leads to Early Detection of Cardiac Tamponade in the Percutaneous intracardiac Intervention
}

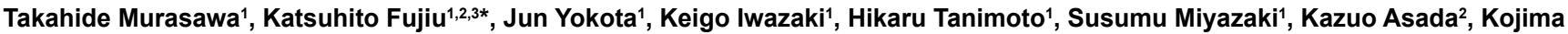 \\ Toshiya2, Yasushi Imai' ${ }^{2}$ Masahiko Sumitani ${ }^{4}$ and Issei Komuro ${ }^{1,2}$
}

${ }^{1}$ The University of Tokyo Hospital, Japan

${ }^{2}$ Department of Cardiovascular Medicine, The University of Tokyo Graduate School of Medicine, Japan

${ }^{3}$ Translational Systems Biology and Medicine Initiative, The University of Tokyo Graduate School of Medicine, Japan

${ }^{4}$ Department of Anesthesiology, The University of Tokyo Graduate School of Medicine, Japan

\begin{abstract}
Background: Cardiac tamponade is one of the major complications which can result from catheter-based cardiac management, and if its detection or treatment is delayed, it can be fatal. Detecting cardiac tamponade in the earliest possible stages is crucial for preventing its development into a life threatening condition.

Methods and results: In this study, an internal blood pressure reading was conducted as part of catheter ablation, cardiac electro physiologic study, and bi-ventricular pacing treatments, and this blood pressure wave form was used along with a FloTrac system to produce a continuous estimate of cardiac output. These data were then retrospectively analyzed to determine whether the FloTrac cardiac output showed changes prior to the onset of declining blood pressure, which is the typical indicator used to diagnose cardiac tamponade. The study was conducted on 213 subjects who were undergoing catheter ablation, cardiac electrophysiologic study, and bi-ventricular pacing treatments. Out of this group, 4 $(1.9 \%)$ were diagnosed with cardiac tamponade. The FloTrac data for all of these cardiac tamponade subjects showed a decline in stroke volume and cardiac output, with the onset of these changes coming an average of $15.5 \pm 6.2$ minutes and $8.7 \pm 1.0$ minutes respectively in advance of the onset of declining blood pressure measurements from the internal blood pressure readings.
\end{abstract}

Conclusion: Continuous cardiac output measurements can be used to detect cardiac tamponade at an earlier stage than continuous internal blood pressure readings, and this could enable accelerated treatment of these complications.

\section{Keywords: Arrhythmia; Ablation; Cardiac tamponade; FloTrac}

\section{Introduction}

In addition topercutaneous coronary intervention for the treatment of angina pectoris and myocardial infarction, percutaneous intracardiac managements for atrial fibrillation such as catheter ablation, closure of atrial and ventricular septal defects and patent foramen ovale, percutaneous heart valvular repair and replacement and left appendage occlusion, as well as bi-ventricular pacing, have started to be performed in recent years. As a result, percutaneous intracardiac procedures have grown more complex and diversified. An especially large portion of these procedures make use of trans septal techniques which require access to the left atrium. Along with this increase in the number of procedures using trans septal techniques, there is a greater risk of patients developing cardiac tamponade conditions in recent years [1]. Cardiac tamponade is defined as the accumulation of a large amount of fluid (usually blood) in the pericardial sac around the heart, which causes a decline in cardiac performance [2]. The physiological requirements are $20-50 \mathrm{ml}$ of fluid, and cardiac tamponade occurs through acute fluid retention from cardiac rupture or chronic accumulation from heart failure or malignant disease [3-5]. In the case of percutaneous intracardiac procedures for the treatment of cardiac rupture, when a sudden influx of blood increases the volume of this cardiac fluid, even as little as $100 \mathrm{ml}$ of fluid accumulation can cause a sudden increase in intracardiac pressure, tachycardia, and deterioration of hemodynamics [4,5]. This is called acute cardiac tamponade, and can be fatal unless it is detected and treated in the early stages [4]. In this study, using the internal arterial pressure and aortic pressure waveforms which are commonly monitored in most cases where percutaneous intracardiac management are implemented, cardiac performance was calculated using a minimally invasive hemodynamic monitoring system: FloTrac Sensor (Edwards Life sciences, Irvine, CA, USA) [6]. This monitoring system enables us to detect stroke volume and cardiac out continuously. We hypothesized that stroke volume and cardiac output can reflect the changes of cardiac performance by cardiac tamponade sensitively, compared to blood pressure more affected by influence of peripheral arterial resistance. This study then considered whether this continuous cardiac performance data could be used as a simple and low-cost system for early detection of cardiac tamponade.

\section{Materials and Methods}

\section{Subjects}

This study was conducted using 213 subjects who received percutaneous cardiac management treatments (including catheter ablation, cardiac electrophysiology study, and bi-ventricular pacing) for arrhythmia and heart failure at the University of Tokyo Hospital between June 2011 and July 2013.

*Corresponding author: Katsuhito Fujiu, Department of Cardiovascular Medicine University of Tokyo, 7-3-1, Hongo, Bunkyo, Tokyo Japan 113-8655, Tel: +81-33815-5411; Fax: +81-3-3818-6673; E-mail: fujiu-tky@umin.ac.jp

Received July 29, 2013; Accepted August 19, 2013; Published August 21, 2013

Citation: Murasawa T, Fujiu K, Yokota J, Iwazaki K, Tanimoto H, et al. (2013) Continuous Cardiac Stroke Volume Monitoring Leads to Early Detection of Cardiac Tamponade in the Percutaneous intracardiac Intervention. J Hypertens 2: 122. doi:10.4172/2167-1095.1000122

Copyright: (c) 2013 Murasawa T, et al. This is an open-access article distributed under the terms of the Creative Commons Attribution License, which permits unrestricted use, distribution, and reproduction in any medium, provided the original author and source are credited. 


\section{Measurements and study design}

A 4Fr sheath was inserted into the right superficial femoral artery, and this arterial line was then connected to a third-generation FloTrac/ Vigileo device (Edwards Life sciences, Irvine, CA, USA). As treatment progressed, this provided a continuous measurement of stroke volume and cardiac output, and at the same time an RMC-4000 Cardio Master (Nihon Kohden, Tokyo, Japan) unit was used for analysis of internal arterial pressure, electrocardiogram, and heart rate. We used the values of continuous cardiac output (CCO) automatically displayed on the FloTrac/Vigileo system averaged over a 20 second rolling period.

\section{Data analysis}

All data were subjected to retrospective analysis. The onset of blood pressure, stroke volume, and cardiac output decline were determined as the point of intersection between two lines, one plotted using the values from the 10 minute period just before the decline and one plotted using the values from the 10 minute period just after the decline started.

\section{Statistical analysis}

All data are expressed as mean $\pm \mathrm{SD}$. When the samples belong to two different classes and have unequal variance, Welch's t-test was used.

\section{Results}

Out of the 213 subjects, acute cardiac tamponade was observed in 4 patients (Table 1). All 4 of these patients received catheter ablation treatments for atrial fibrillation, and cardiac tamponade was diagnosed during the operations and either during the procedures or immediately after, drainage was implemented through pericardiocentesis. Afterward, no further treatments were necessary, and no after-effects were observed. In the remaining 209 patients where cardiac tamponade did not occur, there was no noticeable decline in stroke volume or cardiac output observed during their operations.

In the 4 patients who experienced cardiac tamponade, a comparison of the onset of declines in stroke volume, cardiac output, and arterial pressure showed that the decline of stroke volume and cardiac output preceded the decline of arterial pressure by an average of $15.5 \pm 6.2$ minutes and $8.7 \pm 1.0$ minutes, respectively (Figures $1 \mathrm{a}$ and $1 \mathrm{~b}$ ). This earlier decline of stroke volume values as compared to cardiac output was not proven to be a significant difference, but there was a trend of early onset declines in stroke volume values (Figure 1b). Actually, differences between the onsets of stroke volume drop to the onset of blood pressure drop were 2.7, 3.3, 6.7 and $14.3 \mathrm{~min}$ in case 1, 2, 3 and 4, respectively. These periods might provide more sufficient time for first aid of cardiac tamponade. Here are 2 representative cases (case 1 and 2) in more detail (Figures 2 and 3 ).

For the sake of early detection of cardiac tamponade, direct monitoring of the root of the condition, pericardial effusion, is the theoretical ideal. Observation of pericardial effusion through transthoracic, esophageal, and intracardiac echocardiography are in fact currently in use [1,7]. However, continuous monitoring through trans thoracic echocardiography obstructs catheterization operations, so implementing it during this type of treatment is simply

\begin{tabular}{|c|c|}
\hline no cardiac tamponade & 209 \\
\hline acute cardiac tamponade (during procedure) & $4(1.3 \%)$ \\
\hline acute cardiac tamponade (post procedure) & $0(0.0 \%)$ \\
\hline
\end{tabular}

Table 1: Patients $(n=213)$
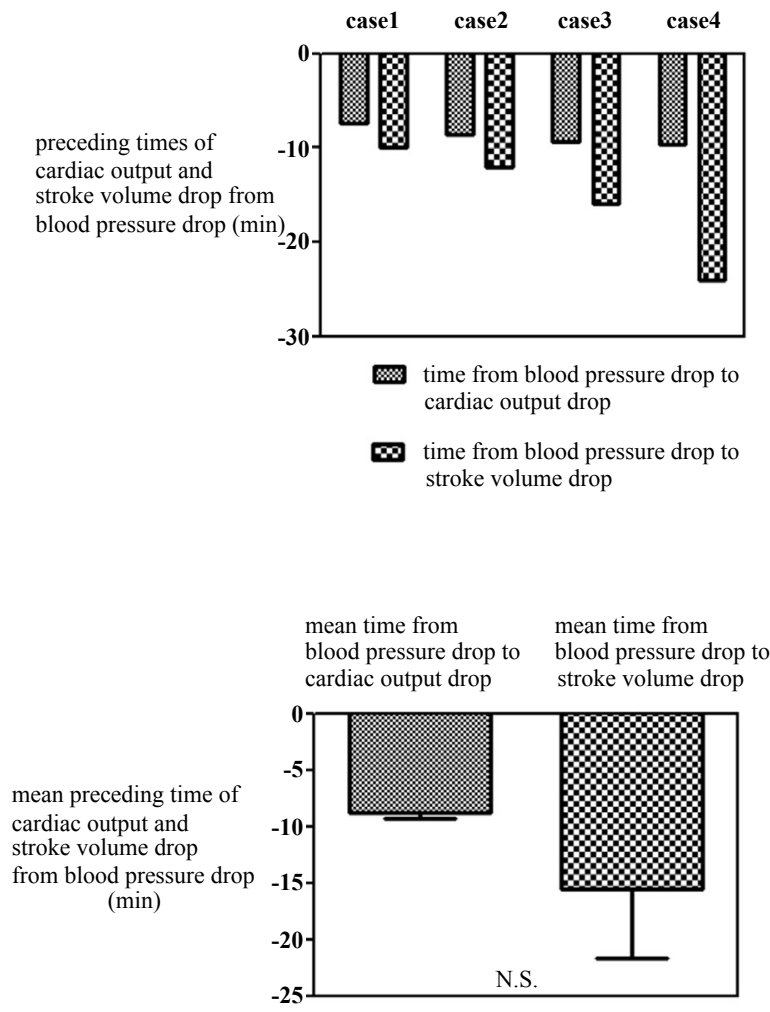

Figure 1: Early onsets of decline of stroke volume and cardiac output before hypotension in acute cardiac tamponade. Onsets of stroke volume and cardiac output decline were analyzed by continuous stroke volume and cardiac output measurement by FloTrac/Vigileo system cardiac performance indicators in cardiac tamponade cases. Degrees to which the decline of stroke volume measurements and cardiac output through FloTrac preceded the onset of arterial pressure decline ( $\mathrm{min}$ ) in four acute cardiac tamponade cases (a). Mean degrees of these four cases to which the decline of stroke volume and cardiac output measurements through FloTrac preceded the onset of arterial pressure decline $(\mathrm{min}) \pm \mathrm{SD}$ (b). N.S.: no significant statistical differences

not possible. Also, continuous monitoring through trans esophageal echocardiography necessitates general anesthetic and the monitoring of an anesthesiologist, so it's not realistic. In addition, although the use of intracardiac echocardiography for continuous monitoring is a possibility, it is an invasive procedure, and the cost can also be problematic. At present, it is possible to cheaply monitor aortic pressure, heartbeat, and right atrial pressure, and these measurements are frequently incorporated into treatment. These parameters have been used for early detection of cardiac tamponade [1]. Concerning aortic pressure measurements, although a decline in aortic pressure is the final indicator of cardiac tamponade in clinical findings, in the early stages of actual cases the blood pressure changes go through various stages. In the initial stage, the sympathetic response to the initial pericardial injury might increase heart rate and blood pressure. On the other hand, bradycardia and hypotension will also occur in the very early stage by cardiac fluid leading to sudden pericardial stretch and vasovagal reflex [1]. In addition, cardiac tamponade provokes adrenergic stimulation and induces the peripheral arterial constriction. As a results of these opposite autonomic nerve activity and adrenergic responses, blood pressure and heart rate were controlled complexity. In this study, the use of a standard arterial line for continuous monitoring of stroke volume provided indicators for the earliest stage detection of cardiac 


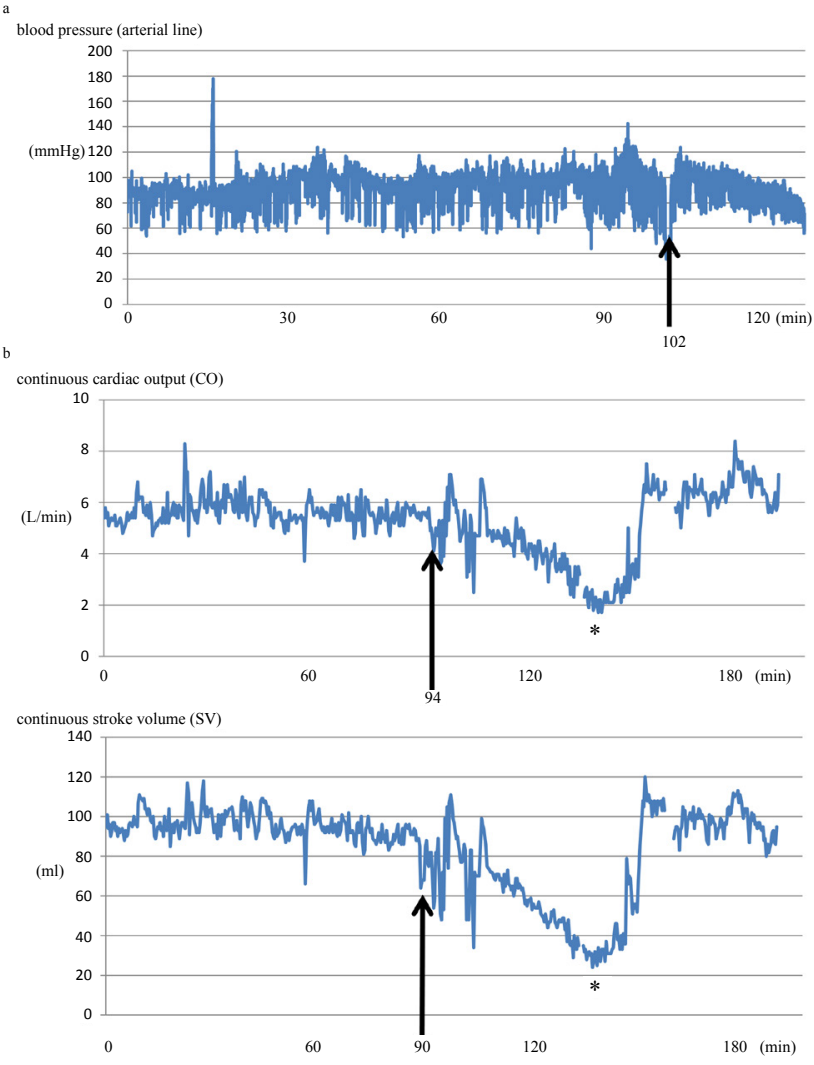

Figure 2: Onset of acute cardiac tamponade during catheter ablation procedures: representative case 1 . Continuous measurements of arterial pressure from the arterial line $(\mathrm{mmHg})(\mathrm{a})$, stroke volume $(\mathrm{ml})(\mathrm{b})$ and cardiac output $(\mathrm{L} / \mathrm{min})(\mathrm{c})$ estimated by the FloTrac system from the arterial pressure waveform. Arrows show the onsets of decline of these parameters during acute cardiac tamponade. Asterisk shows timing of drainage of cardiac fluid.

tamponade. This is not comparable to intracardiac echocardiography in terms of early detection of pericardial fluid, but stroke volume and cardiac output values, more so than monitoring arterial pressure and heart rate, seem to be a more direct reflection of the state of patients' heart function in the complex condition like cardiac tamponade. In addition, stroke volume measurements showed a tendency toward greater sensitivity, and this is thought to be because they might be lessaffected by activation of the autonomic nerves resulting from cardiac rupture and cardiac tamponade, compared to heart rate and blood pressure.

About other detection methods for cardiac tamponade by simple and common methods, cardiac silhouette evaluation by chest radiography has been reported as another low cost method for early stage detection $[8,9]$. According to these reports, if there is a large volume of accumulated pericardial effusion, the cardiac silhouette will show a distortion in a shape similar to a water bottle, and a distinctive image called an epicardial halo sign or fat pad sign will show up $[8,9]$. In our experiences with chest radiography, the pulsations of the cardiac silhouette get smaller called "locking heart" in cases of acute cardiac tamponade. In this way, diagnosis through radiography is likely a possibility. However, it is difficult to detect a cardiac tamponade by these radiographic signs because recognition of these signs requires sufficient clinical trainings and experiences. Actually, in the case of our 4 patients with cardiac tamponade, we could not find any radiographic abnormal signs before the onset of blood pressure decline. However, in real-life applications, interpreting the findings of this radiography requires training and experience. Also, if we consider this study's 4 patients with cardiac tamponade, it would not have been possible to diagnose the condition through chest radiography prior to the onset of blood pressure decline. As a result of the factors above, it is our expectation that the use of continuous cardiac stroke volume monitoring during percutaneous invasive heart management procedures will be useful for early stage detection of acute cardiac tamponade. Moreover, this continuous cardiac output monitoring do not require any additional invasive setup if arterial pressure monitoring from sheath in an artery or arterial line is a routine method.

\section{Study limitations}

The limitations of this study include that it was a single-center retrospective study with no long-term follow. Larger and prospective studies are needed to further assess the clinical feasibility of continuous cardiac performance monitoring to predict cardiac tamponade in percutaneous heart managements. For example, prospective studies whether stroke volume or cardiac output decline which fulfills defined criterias without a blood pressure drop can predict cardiac tamponade and whether it is useful for rapid first aid and results in decreasing severe development of cardiac tamponadeare candidates of future study design.
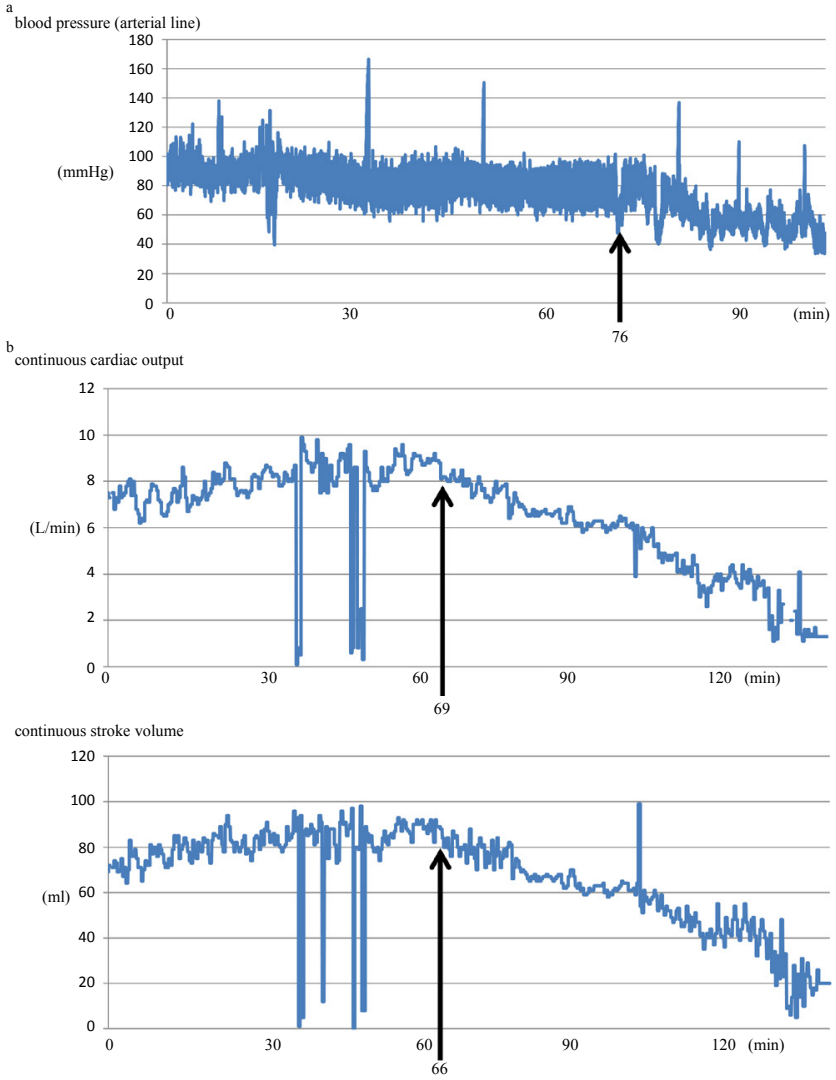

Figure 3: Onset of acute cardiac tamponade during catheter ablation procedures: representative case 2 . Continuous measurements of arterial pressure from the arterial line $(\mathrm{mmHg})(\mathrm{a})$, stroke volume $(\mathrm{ml})(\mathrm{b})$ and cardiac output (L/min) (c) estimated by the FloTrac system from the arterial pressure waveform. Arrows show the onsets of decline of these parameters during acute cardiac tamponade. 
Citation: Murasawa T, Fujiu K, Yokota J, Iwazaki K, Tanimoto H, et al. (2013) Continuous Cardiac Stroke Volume Monitoring Leads to Early Detection of Cardiac Tamponade in the Percutaneous intracardiac Intervention. J Hypertens 2: 122. doi:10.4172/2167-1095.1000122

\section{Acknowledgements}

We gratefully acknowledge Yoko Maruyama, Harumi Nakatani, Reiko Fujii, Kaori Toriumi, Keiko Yoshizawa, Chiori Higuchi, Noriko Takahashi, Kyoko Endo, Kayomi Shimozono, Katsumi Shigaura for their excellent technical assistance.

\section{References}

1. Holmes DR Jr, Nishimura R, Fountain R, Turi ZG (2009) latrogenic pericardia effusion and tamponade in the percutaneous intracardiac intervention era. JACC Cardiovasc Interv 2: 705-717.

2. Rose E (1884) Herztamponade. Dtsch Z Chir 13: 329-410.

3. Holt JP, Rhode EA, Kines H (1960) Pericardial and ventricular pressure. Circ Res 8: 1171-1181.

4. Spodick DH (2003) Acute cardiac tamponade. N Engl J Med 349: 684-690
5. Goldstein JA (2004) Cardiac tamponade, constrictive pericarditis, and restrictive cardiomyopathy. Curr Probl Cardiol 29: 503-567.

6. Manecke GR (2005) Edwards FloTrac sensor and Vigileo monitor: easy, accurate, reliable cardiac output assessment using the arterial pulse wave. Expert Rev Med Devices 2: 523-527.

7. Ren JF, Marchlinski FE (2010) Early detection of iatrogenic pericardial effusion importance of intracardiac echocardiography. JACC Cardiovasc Interv 3: 127.

8. Carsky EW, Mauceri RA, Azimi F (1980) The epicardial fat pad sign: analysis of frontal and lateral chest radiographs in patients with pericardial effusion. Radiology 137: 303-308.

9. Maisch B, Seferovic PM, Ristic AD, Erbel R, Rienmuller R, et al. (2004) Guidelines on the diagnosis and management of pericardial diseases executive summary; The Task force on the diagnosis and management of pericardial diseases of the European society of cardiology. Eur Heart J 25: 587-610. 